\title{
A glossary of television-related symptoms in psychosis
}

\author{
M. G. Smyth
}

\begin{abstract}
Although the attribution of psycholic symptoms to the television is widespread, there is little liferature on the subject and one might surmise that the issue is considered so mundane as not to mertt further interest.

in so far as 'reality' is a critical problem in psychosis, television can constitute a particular source of confusion. 'Recilty' can be fudged by this medlum for us all, with editing, propaganda, and the spectrum of front line live reports, 'real life' re-enactments, and drama.
\end{abstract}

\section{The study}

The aim of the present report was to describe the range of psychotic symptoms attributed to the television in a sample of people with schizophrenia and in so doing, look for any patterns of attribution. The enquiry was not empirically designed, and was essentially qualitative. The sample of 18 patients with diagnosed schizophrenia (for whom the television was problematic) was recruited over two years, through routine clinical contact mainly at out-patient clinics. Their range of psychotic symptomatology was elicited through relevant Present State Examination questions, and a semi-structured questionnaire regarding described relationships between symptoms and television.

\section{Findings}

The sample comprised 13 men and five women. By definition, all subjects suffered from delusions of reference associated with the television. This was described as a passive influence by all subjects, but three additionally described a reciprocal relationship whereby they could exert influence (for example, Case 4 described the manner in which individuals on television absorbed her influences beneficially; Case 3 described applause as signifying that their attempts to disseminate peace through constant prayer before the television, had been effective and acknowledged).

The usual pattern was for subjects readily to identify two or three specific symptoms while dismissing others. Seven subjects attributed 'thought insertion' directly to the television (for example, newscasters were putting thoughts into the patient's brain through the movement of their teeth-separate and distinct from spoken material, in another case, disturbing thoughts of stabbing (her cats and partner) were being implanted in the patient's mind secretly).

Two subjects described 'thought echo' (e.g. "They are having the exact same thoughts as me - it is beyond coincidence"). Four subjects ascribed 'thought broadcasting' to the television (e.g. "Actors can hear what I am thinking. straight away": "I worry about how much of my thoughts they can hear on chat shows"). There were three examples of 'thought withdrawal' (e.g. "when I think of something, the character takes it up straight away, and I feel it leaving me") and two cases of 'thoughts being read' (e.g. "they read my thoughts even with all my jargon in it").

Auditory hallucinations were attributed to the television. Three subjects explained all of their hallucinations as coming from the television, but this was unusual. It was more common for certain specific 'voices' to be identified with the television (while not other voices') and six cases described auditory hallucinations which were not felt to have any relationship to watching television (despite having other television-associated delusions of reference). The hallucinations attributed to the television were all command or derogatory in content and in the second person.

Further enquiry focused on aspects of television watching that could be relevant to the occurrence of symptoms. The following items were irrelevant in all cases: colour/black and white; live/recorded broadcasts; interference; teletext; and the use of remote control devices. Relevant items were those of: different channels; avoidance behaviour; watching alone or in the presence of others; and programme type.

Two subjects experienced problems only with certain channels (Channel 4 in one case, and both BBC 1 and 2, in another). A further subject changed channels frequently in an attempt to stem hallucinations.

The majority (16) were quite aware of differences between watching alone, or in company. Eleven found watching in the company of others somewhat protective and less disturbing than watching alone (five reported the opposite). 
Ten subjects had developed an established pattern of avoidance behaviour (e.g. not watching alone; not watching the news). The inability to avoid television on the ward during an in-patient stay was described as frustrating by two from this group.

The most striking findings arose in respect of programme type, where only one subject did not discriminate. Subjects were asked to rank the programmes which they associated with greater and lesser disturbance. The most problematic programmes were (in decreasing order), news broadcasts, advertisements, and game shows. Programmes associated with least disturbance were: nature programmes, sports, documentaries, and music. Programmes with reported disturbance intermediate between these extremes were: comedy, soaps, and drama films.

\section{Comment}

The study was not empirical and particular methodological difficulties would arise were this to be attempted on the basis of questionnaire alone (such as refining specific programme types, controlling for exposure, and visual combined with auditory stimuli). No claims can be made in terms of the frequency or range of symptoms (and fundamentally all involve explanatory delusions of reference). Cramer et al (1989) found that people with schizophrenia displayed deficits and deviant responses in judging the emotional states of others. The degree of structure in external sensory stimulation has been found to influence the occurrence of hallucinations in schizophrenia (Margo et al. 1981).

Why were news programmes so disturbing? News is usually serious, important and novel. The absence of varying expressiveness in the facial manner of news readers (i.e. lack of clues) could be problematic for patients with schizophrenia who have impaired social sensitivity. Additionally, some patients described the particular emphatic and jerky head movement of broadcasters as conveying additional and personal meaning.

Regarding advertisements, it was those adverts which incorporated tangential or lateral thinking in their design which were found difficult, e.g. sudden scene changes as in "Time for a sharp exit" (Harp) and surrealistic adverts (e.g. Guinness). By contrast, nature programmes seldom involve people, and the commentary is friendly and explanatory. Documentaries also incorporate explanation and an understandable sequence of exposition. Audience applause and laughter (on game shows, for instance) were often cited as being somehow mocking, confusing or derogatory.

It is often difficult to diagnose the presence of psychosis with certainty, particularly in emergent schizophrenia, and in one-off assessments. I was struck by the enthusiasm of patients to discuss in detail symptoms which revolved around the television, and the degree to which. perhaps feeling understood and with an improved therapeutic alliance, more forthcoming mental state examinations were possible. Television seemed to be a neutral and less invasive topic than others. I would recommend psychiatrists in training to consider extending their questioning around this topic, where appropriate, in order to clarify and refine elicited psychopathology.

\section{References}

Cramer, P.. Weegmann, M. \& O'Neill, M. (1989) Schizophrenia and the perception of emotions. How accurately do schizophrenics judge the emotional states of others? British Journal of Psychiatry, 165, 225-228.

Margo, A., Hemsley, D. R. \& Slade, P. D. (1981) The effects of varying auditory input on schizophrenic hallucinations. British Journal of Psychiatry. 139. 122-127.

M. G. Smyth, Academic Consultant, Department of Psychiatry, University of Birmingham, 71 Fentham Road, Birmingham B23 6AL 